TITLE:

\title{
Possibility of direct determination of the quantum phase of continua utilizing the phase of lasers
}

$\operatorname{AUTHOR}(S)$ :

Nakajima, T

\section{CITATION:}

Nakajima, T. Possibility of direct determination of the quantum phase of continua utilizing the phase of lasers. PHYSICAL REVIEW A 2000, 61(4): 041403.

ISSUE DATE:

2000-04

URL:

http://hdl.handle.net/2433/50412

RIGHT:

Copyright 2000 American Physical Society 


\title{
Possibility of direct determination of the quantum phase of continua utilizing the phase of lasers
}

\author{
Takashi Nakajima \\ Institute of Advanced Energy, Kyoto University, Gokasho, Uji, Kyoto 611-0011, Japan
}

(Received 25 August 1999; published 17 March 2000)

\begin{abstract}
We show that the quantum phase of continua can be directly determined utilizing the phase of linearly/ circularly polarized lasers. With this method the phase difference of continua with opposite as well as same parities can be obtained from the phase lag of the angle-resolved photoelectron signal with respect to the relative phase of lasers. For illustration, the proposed method is specifically applied to the $\mathrm{Na}$ atom.
\end{abstract}

PACS number(s): 32.80.Qk, 32.80.Rm, 33.80.Rv

One of the ways of controlling the light-matter interaction is to manipulate the phase of lasers by which a destructive or constructive interference is induced between different transition paths to the same final state [1-17]. Experimentally this is often realized using a fundamental and its third-harmonic $(\omega-3 \omega$ scenario) $[2-7]$ or second-harmonic $(\omega-2 \omega$ scenario) waves [8-11]. For a bound-bound transition, no interference occurs with the $\omega-2 \omega$ scenario because of the parity selection rule, while with the $\omega-3 \omega$ scenario, an interference takes place and a transition strength can be manipulated by controlling the relative phase of two laser fields. For a boundcontinuum transition, the phase-dependent interference can be observed even with the $\omega$ - $2 \omega$ scenario as long as the photoelectron or photodissociation signals are angle resolved $[8,10]$. Needless to say, the total ionization signal does not exhibit any phase-sensitive interference in such a case. As for the $\omega$ - $3 \omega$ scenario, the phase-dependent interference can be observed for both angle-resolved and angle-integrated ionization signals [13].

It is well known that a photoelectron angular distribution (PAD) contains a lot of information on transition amplitudes and the difference of the phase shifts of wave functions, and in principle these quantities could be indirectly determined by measuring PADs $[18,19]$ and fitting theoretical curves to them. However, experimental errors could lead to the large uncertainty of the extracted phase shifts after fittings. More importantly, apart from such an experimental uncertainty, there is a serious problem in the use of the PAD technique for determining phase shifts; for example, for nonresonant two-photon ionization of alkali-metal atoms from the $s$ state, there are two fitting parameters, while three parameters are theoretically required for the unambiguous determination of phase shifts as extensively discussed in Ref. [18]. If the spinorbit interaction is negligible, the number of theoretical parameters is reduced from three to two, and the phase shifts could be uniquely determined from the PAD data, in principle. On the other hand, if the spin-orbit interaction is not negligible, three independent physical quantities cannot be extracted from the two fitted quantities, i.e., the PAD technique faces a serious problem.

The potential use of the phase of lasers for the determination of the phase difference of continua has been briefly mentioned by Elliott and co-workers [8], but a straightforward application of the $\omega-2 \omega$ scenario instead of a singlecolor ionization does not improve the situation for that purpose: In the presence of two laser fields, it is quite essential to know the precise values of laser intensities and the ratios of all transition amplitudes, if the standard method mentioned above is to be applied to extract the values of phase difference. The use of two laser fields simply introduces additional complexity, leading to the even lower reliability of the fitted quantities.

The purpose of this paper is to show that the quantum phase of continua can be directly and precisely determined with the use of the phase of lasers based on the $\omega$ - $2 \omega$ scenario. Especially, the proposed method allows us to determine the phase difference between continua with different parities; for example, $s$ and $p$ continua, which cannot be measured otherwise. As explained later on in this paper, this would become possible by a careful choice of laser polarization and also by isolating particular partial waves of the continua of interest through angle-resolved photoelectron detection. In contrast to the conventional PAD measurement, the phase difference is observed as a phase lag $[5,15]$ with this method with respect to the relative phase of two lasers. It is interesting to point out that, when this method is applied to light alkali-metal atoms such as $\mathrm{Li}, \mathrm{Na}$, or $\mathrm{K}$, the ratio of the radial matrix elements does not play a role in the determination of phase difference. For heavier alkali-metal atoms, such as $\mathrm{Rb}$ or $\mathrm{Cs}$, the spin-orbit interaction plays an important role, and more careful consideration is necessary. Nevertheless, it is shown that the direct determination of the phase difference of continua is possible, in principle, even in such a case.

We restrict ourselves to the case of an alkali-metal atom, and start with the description of a continuum. For a given wave vector $\vec{k}$ and magnetic quantum number $m_{s}$ of the spin of the outgoing photoelectron, the continuum state is expanded using partial waves:

$$
\left|\vec{k} ; m_{s}\right\rangle=\sum_{l, m_{l}} a_{l m_{l}}\left|\vec{k} ; l \frac{1}{2} m_{l} m_{s}\right\rangle
$$

where $\quad a_{l m_{l}}=4 \pi i^{l} e^{-i \delta_{l}} Y_{l m_{l}}^{*}(\Theta, \Phi) \quad$ and $\quad\left|\vec{k} ; l \frac{1}{2} m_{l} m_{s}\right\rangle$ $=\left|l m_{l}\right\rangle\left|\frac{1}{2} m_{s}\right\rangle$. For an alkali-metal atom, the phase shift $\delta_{l}$ of the $l$ partial wave is a sum of the Coulomb phase shift given by $\arg [\Gamma(l+1-i / \sqrt{W})]-\pi l / 2$, where the kinetic energy of the photoelectron, $W$, is in Rydberg units, and the shift is due to the quantum defect $\pi \mu_{l}$ where $\mu_{l}$ is the extrapolated quantum defect for the bound $l$ state. $Y_{l m}$ is the spherical 
harmonics, and $(\Theta, \Phi)(\equiv \Omega)$ defines the direction of the outgoing photoelectron with respect to the quantization axis. It should be understood that the quantization axis is taken along the polarization direction for linearly polarized light and the propagation direction for circularly polarized light, respectively.

In the presence of two laser fields with amplitudes $\varepsilon_{\omega}$ and $\varepsilon_{2 \omega}$ for frequencies $\omega$ and $2 \omega$, and relative phase $\phi$, the total laser field is written as $E(t)=\left(\varepsilon_{\omega} e^{i \omega t}+\varepsilon_{2 \omega} e^{i(2 \omega t+\phi)}\right)+$ c.c. Assuming that an initial state is unpolarized and the spin of photoelectrons is not resolved, the differential ionization rate is written in the following form:

$$
\begin{aligned}
\frac{d \sigma}{d \Omega}= & \sum_{m_{s}} \mid \sum_{l m_{l}} a_{l m_{l}}^{*}(-1)^{l-1 / 2+m_{j}} \sqrt{2 j+1}\left(\begin{array}{ccc}
l & s & j \\
m_{l} & m_{s} & -m_{j}
\end{array}\right) \\
& \times\left.\left\langle\vec{k} ;\left(l \frac{1}{2}\right) j m_{j}\left|e^{i \phi} D_{q}+D_{q}^{(2)}\right|\left(l^{\prime} \frac{1}{2}\right) j^{\prime} m_{j}^{\prime}\right\rangle\right|^{2}
\end{aligned}
$$

where $D_{q}$ and $D_{q}^{(2)}$ are the single- and two-photon dipole operators, respectively, which are nothing but the $q$ th spherical components of the dipole moments $r_{q}$ and $r_{q}^{(2)}$ multiplied by some appropriate conversion coefficients $C_{2 \omega}$ and $C_{\omega}$, i.e., $D_{q}=C_{2 \omega} r_{q}$ and $D_{q}^{(2)}=C_{\omega} r_{q}^{(2)}$. If the dipole moments $r_{q}$ and $r_{q}^{(2)}$ are given in atomic units and the laser intensities $I_{2 \omega}$ and $I_{\omega}$ in $\mathrm{W} / \mathrm{cm}^{2}$, they are given by $C_{2 \omega}=0.767 \sqrt{I_{2 \omega}}$ and $C_{\omega}=2.05 \times 10^{-9} I_{\omega}$, respectively [13]. Now we assume that the initial state is the ground state, i.e., $s_{1 / 2}$ state. In such a case, if both fields are linearly polarized, Eq. (2) becomes

$$
\begin{aligned}
\frac{d \sigma}{d \Omega} \propto & -i \frac{\sqrt{3}}{3} C_{2 \omega} Y_{10}\left(R_{1}+2 R_{2}\right) e^{i\left(\delta_{p}+\phi\right)} \\
& +C_{\omega}\left\{\frac{1}{3} Y_{00}\left(R_{1}^{(2)}+2 R_{2}^{(2)}\right) e^{i \delta_{s}}\right. \\
& \left.-\frac{2 \sqrt{5}}{75} Y_{20}\left(5 R_{3}^{(2)}+R_{4}^{(2)}+9 R_{5}^{(2)}\right) e^{i \delta_{d}}\right\}\left.\right|^{2} \\
& +\mid i \frac{2 \sqrt{3}}{3} C_{2 \omega} Y_{11}\left(R_{1}-R_{2}\right) e^{i\left(\delta_{p}+\phi\right)} \\
& -\left.\frac{\sqrt{30}}{75} C_{\omega} Y_{21}\left(-5 R_{3}^{(2)}-R_{4}^{(2)}+6 R_{5}^{(2)}\right) e^{i \delta_{d}}\right|^{2},
\end{aligned}
$$

where $R_{1}, R_{2}$ are single-photon radial matrix elements from $s_{1 / 2}$ to $k p_{1 / 2}$ and $k p_{3 / 2}$, respectively, and $R_{a}^{(2)}$ $(a=1,2, \ldots, 5)$ are effective two-photon radial matrix elements defined, respectively, as $s_{1 / 2} \rightarrow p_{1 / 2} \rightarrow k s_{1 / 2}, s_{1 / 2} \rightarrow p_{3 / 2}$ $\rightarrow k s_{1 / 2}, \quad s_{1 / 2} \rightarrow p_{1 / 2} \rightarrow k d_{3 / 2}, \quad s_{1 / 2} \rightarrow p_{3 / 2} \rightarrow k d_{3 / 2}, \quad$ and $\quad s_{1 / 2}$ $\rightarrow p_{3 / 2} \rightarrow k d_{5 / 2}$. If, on the other hand, both fields are right circularly polarized, Eq. (2) becomes

$$
\begin{aligned}
\frac{d \sigma}{d \Omega} \propto & -i \sqrt{3} C_{2 \omega} Y_{11} R_{2} e^{i\left(\delta_{p}+\phi\right)}-\left.\frac{\sqrt{30}}{5} C_{\omega} Y_{22} R_{5}^{(2)} e^{i \delta_{d}}\right|^{2} \\
& +\mid-i \frac{\sqrt{3}}{3} C_{2 \omega} Y_{11}\left(R_{1}+2 R_{2}\right) e^{i\left(\delta_{p}+\phi\right)} \\
& -\left.\frac{\sqrt{30}}{75} C_{\omega} Y_{22}\left(10 R_{3}^{(2)}+2 R_{4}^{(2)}+3 R_{5}^{(2)}\right) e^{i \delta_{d}}\right|^{2} \\
& +\mid-i \frac{\sqrt{6}}{3} C_{2 \omega} Y_{10}\left(R_{1}-R_{2}\right) e^{i\left(\delta_{p}+\phi\right)} \\
& +\left.\frac{\sqrt{30}}{75} C_{\omega} Y_{21}\left(5 R_{3}^{(2)}+R_{4}^{(2)}-6 R_{5}^{(2)}\right) e^{i \delta_{d}}\right|^{2} .
\end{aligned}
$$

It should be noted that, in Eqs. (3) and (4), the $j$ dependence of the phase shifts $\delta_{l}$ has been ignored, since, even for Cs, such a dependence is known to be very small compared with that of the radial matrix elements [20].

Based on Eqs. (3) and (4), we consider two cases depending on the strength of spin-orbit interaction. As a first case, assume that the spin-orbit interaction is so weak that the radial matrix elements have approximately no $j$ dependence, i.e., $R_{1} \simeq R_{2}, R_{1}^{(2)} \simeq R_{2}^{(2)}$, and $R_{3}^{(2)} \simeq R_{4}^{(2)} \simeq R_{5}^{(2)}$. Such an assumption is valid for $\mathrm{Li}, \mathrm{Na}$, and $\mathrm{K}$. In this case, the last terms in both Eqs. (3) and (4) disappear. It can be seen from Eq. (3) that the angle-resolved photoelectron signal contains information on the phase shifts of $\delta_{s}, \delta_{p}$, and $\delta_{d}$. From an experimental point of view, however, it is not as easy as it appears to determine these quantities, since other free parameters for fittings, i.e., laser intensities and radial matrix elements, are not precisely known, in general. It is interesting to point out that the situation becomes much simpler if we focus on the photoelectron signal emitted to some particular direction. For example, in this particular case, the photoelectron signal emitted to the angles $\Theta=\cos ^{-1}(1 / \sqrt{3})$ and $\pi$ $-\cos ^{-1}(1 / \sqrt{3})$ contains a phase-dependent factor given by $\sin \left(\delta_{p}-\delta_{s}+\phi\right)$, since $Y_{20}$ becomes zero at this $\Theta$. This means that, by measuring the phase lag of the photoelectron signal with respect to the relative phase of lasers $\phi$ at this $\Theta$ (magic angle), the phase difference $\delta_{p}-\delta_{s}$ could be directly and precisely determined. It should be emphasized that this phase lag can be experimentally determined, independent of the values of the radial matrix elements and laser intensities $I_{\omega}$ and $I_{2 \omega}$. Thus, the key to the direct determination of the phase difference is to focus on the photoelectron signal emitted to the magic angle where only a single pair of the partial waves contributes to the signal, and measure the phase lag as a function of the relative phase $\phi$ of lasers. With this particular measurement, however, the phase difference $\delta_{p}-\delta_{d}$ or $\delta_{s}-\delta_{d}$ cannot be determined. Now we consider Eq. (4). The advantage of utilizing circularly polarized light is that we can isolate the other pair of partial waves, which are $p$ and $d$ waves, respectively, for the single- and the two-photon ionization from the $s_{1 / 2}$ ground state. Thus, by using circularly polarized light, the phase difference $\delta_{p}-\delta_{d}$ can be determined. It should be clear at this point that the use of linearly and circularly polarized light is complementary. 
As a second case, assume that the spin-orbit interaction is not negligible. Heavier alkali-metal atoms, $\mathrm{Rb}$ and $\mathrm{Cs}$, fall into this category. In this case, the last terms in Eqs. (3) and (4) cannot be ignored anymore. It is clear from Eq. (4) that this does not cause a serious problem for the case of circularly polarized light. That is not the case, however, for the linearly polarized light, since even at the magic angle the contribution of the $d$ partial wave cannot be ignored for determining $\delta_{p}-\delta_{s}$. In order to correlate the strength of spinorbit interaction with the additional phase lag due to the nonvanishing $d$ wave, we define generalized perturbation functions $x_{1}$ and $x_{2}$ for single- and two-photon processes, respectively, as

$$
\begin{gathered}
x_{1}=\frac{2 R_{2}+R_{1}}{R_{2}-R_{1}}, \\
x_{2}=\frac{5 R_{3}^{(2)}+R_{4}^{(2)}+9 R_{5}^{(2)}}{5 R_{3}^{(2)}+R_{4}^{(2)}-6 R_{5}^{(2)}},
\end{gathered}
$$

and

$$
w=\frac{5\left(R_{1}^{(2)}+2 R_{2}^{(2)}\right)}{5 R_{3}^{(2)}+R_{4}^{(2)}+9 R_{5}^{(2)}} .
$$

Note that all of these parameters $x_{1}, x_{2}$, and $w$ are functions of photoelectron energy. Obviously, if the spin-orbit interaction is weak, $x_{1}$ and $x_{2}$ tend to take large values. $w$ is a kind of ratio of "averaged" two-photon radial matrix elements into $s$ and $d$ waves. Using these quantities and Eq. (3), the $\phi$-dependent modulation $M$ of the photoelectron signal emitted to the magic angle $\Theta=\cos ^{-1}(1 / \sqrt{3})$ is obtained, after some algebra, as

$$
M \propto \sin \left(\delta_{p}-\delta_{s}+\phi\right)+\frac{2 \sqrt{2}}{w x_{1} x_{2}} \sin \left(\delta_{p}-\delta_{d}+\phi\right) .
$$

Equation (8) means that, given the quantities $w, x_{1}, x_{2}$, and $\delta_{p}-\delta_{d}$, which have been determined by using circularly polarized light, the phase difference $\delta_{p}-\delta_{s}$ can be determined from the measurement by linearly polarized light, with a correction due to the spin-orbit interaction. In order to make such a correction, however, these values must be known in advance, which itself would be an experimental as well as a theoretical challenge. This problem might be avoided, at least in principle, by the use of polarized atoms in the $m_{j}$ $=1 / 2(-1 / 2)$ ground state and the detection of spin-up (-down) photoelectrons. Then, only the first (second) term of Eq. (3) contributes to the photoelectron signal, and by detecting photoelectrons at the magic angle (any angle), the phase difference $\delta_{p}-\delta_{s}\left(\delta_{p}-\delta_{d}\right)$ can be unambiguously determined, even in the presence of strong spin-orbit interaction. In other words, in the presence of strong spin-orbit interaction, not only $\delta_{p}-\delta_{s}$ but also $\delta_{p}-\delta_{d}$ can be directly determined using linearly polarized light only, at the expense of a more complicated experimental setup.

A natural question that would come to mind is "how do we define the reference for the phase?" An obvious but not

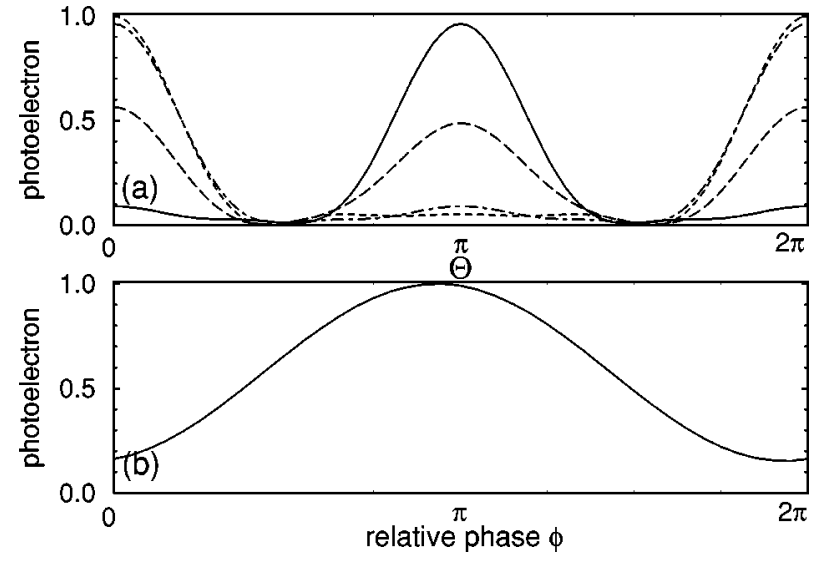

FIG. 1. (a) Variation of the PAD of $\mathrm{Na}$ at $\phi=0$ (solid), $\pi / 3$ (long-dashed), $2 \pi / 3$ (dotted), and $\pi$ (dot-dashed) under two color fields. (b) Variation of the photoelectron signal at $\Theta$ $=\cos ^{-1}(1 / \sqrt{3})$ under two color fields as a function of the relative phase $\phi$. Both lasers are linearly polarized.

necessarily the simplest way is to make use of the PAD of hydrogen, where the quantum phase involved is nothing but a Coulomb phase shift, and use it as a reference to determine the relative phase of lasers. This would be particularly useful for circularly polarized light, since the harmonic generation technique employed for the calibration of the relative phase of linearly polarized lasers $[7,11]$ cannot be immediately applied for circularly polarized lasers. An excited state, for example, the $2 s_{1 / 2}$ state of hydrogen, might be used for calibration, since the photon energies of the lasers might not be sufficient to ionize a hydrogen atom in the ground state. For heavier alkali-metal atoms ( $\mathrm{Rb}$ and $\mathrm{Cs})$, it might be sufficient to calibrate the phase of lasers only for linear polarization, since both $\delta_{p}-\delta_{s}$ and $\delta_{p}-\delta_{d}$ can be determined by linearly polarized light, in principle, based on the argument described above.

Figure 1(a) shows a theoretical PAD of $\mathrm{Na}$ by linearly polarized lasers, based on the single-channel quantum-defect

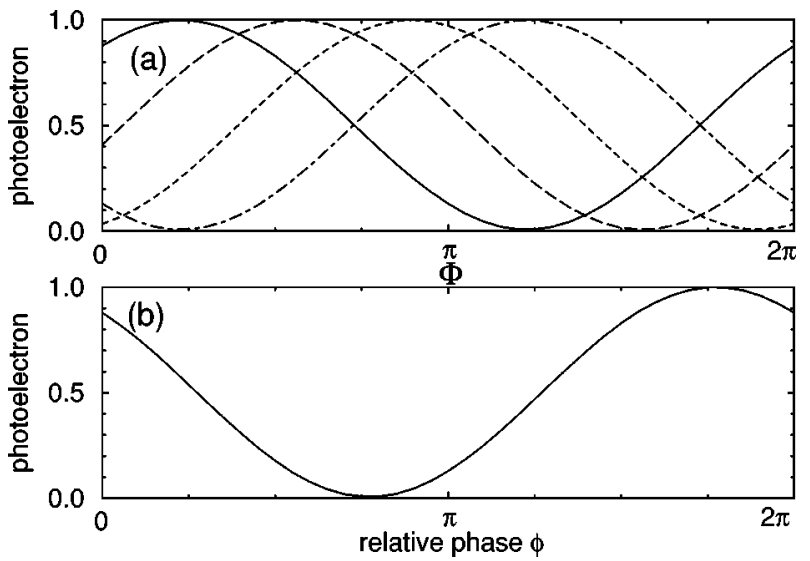

FIG. 2. (a) Variation of the photoelectron signal of $\mathrm{Na}$ at $\Theta=\pi / 2$ as a function of $\Phi$ at four values of the relative phase $\phi=0$ (solid), $\pi / 3$ (dashed), $2 \pi / 3$ (dotted), and $\pi$ (dot-dashed) under two color fields. (b) Variation of the photoelectron signal at $(\Theta, \Phi)=(\pi /$ $2,0)$ as a function of relative phase $\phi$. Both lasers are circularly polarized. 
theory, at the final-state energy of $W=0.0278$ Ry. A significant variation of the PAD is seen as $\phi$ is varied from 0 to $\pi$. We show in Fig. 1(b) the variation of the photoelectron signal emitted to the magic angle $\Theta=\cos ^{-1}(1 / \sqrt{3})$, where the $\phi$-dependent modulation comes from the factor $\sin \left(\delta_{p}-\delta_{s}\right.$ $+\phi)$ as explained before. This way the phase difference of continua can be directly determined from the phase lag, which is insensitive to the ratio of the radial matrix elements or the laser intensities employed. A similar calculation has been performed for circularly polarized lasers, as shown in Fig. 2. With the single-color photoionization by circularly polarized light, the PAD, of course, does not depend on the angle $\Phi$ that defines the azimuthal angle around the propagation axis of the laser. Under the simultaneous action of two lasers with well-defined relative phase, however, the photoelectron signal does depend on the angle $\Phi$ as well as $\Theta$, as shown in Fig. 2(a). The variation of the photoelectron signal to the particular direction defined by $(\Theta, \Phi)=(\pi / 2,0)$ is shown in Fig. 2(b). Again, this result implies that the phase difference $\delta_{p}-\delta_{d}$ can be directly determined from the phase lag.

In summary, we have shown that the phase difference of continua can be directly determined utilizing the phasecontrolled lasers. Although we have specifically applied this method to the alkali-metal atom, it is apparent that the method should work for any other atom as well, as long as the single-active-electron approximation is valid. The most important feature of the proposed method is that the determination of the phase difference is insensitive to the values (ratios) of the radial matrix elements, which would be of great importance if the phase differences were indirectly determined from the PAD. In order to isolate a particular pair of continuum states of interest, it is essential to employ an initial state with proper symmetry and lasers with appropriate polarizations. For the purpose of reaching the continua that are not accessible from the ground state by the single- or two-photon ionization, the initial state might be an excited state. Although the application of this technique for heavier alkali-metal atoms ( $\mathrm{Rb}$ and $\mathrm{Cs}$ ) would require an elaborate experimental setup, i.e., polarized atoms in the ground state and spin-resolved detection of photoelectrons, the phasecontrolled lasers could still be used to determine the phase difference of continua of heavy alkali-metal atoms without ambiguity, since the normal PAD technique does not work for them [18] due to the reason explained in the beginning of this paper. In spite of the technical difficulty, especially for heavy atoms, the proposed method is free from such a problem.

The author acknowledges useful discussions with Professor P. Lambropoulos, Professor M. Kawasaki, Professor I. Shimamura, and Professor D. Charalambidis.
[1] M. Shapiro, J.W. Hepburn, and P. Brumer, Chem. Phys. Lett. 149, 451 (1988).

[2] C. Chen, Y.-Y. Yin, and D.S. Elliott, Phys. Rev. Lett. 64, 507 (1990); 65, 1737 (1990).

[3] N.E. Karapanagioti, D. Xenakis, D. Charalambidis, and C. Fotakis, J. Phys. B 29, 3599 (1996).

[4] S.-M. Park, S.-P. Lu, and R.J. Gordon, J. Chem. Phys. 94, 8622 (1991).

[5] L. Zhu, K. Suto, J.A. Fiss, R. Wada, T. Seideman, and R.J. Gordon, Phys. Rev. Lett. 79, 4108 (1997).

[6] X. Wang, R. Bersohn, K. Takahashi, M. Kawasaki, and H.-L. Kim, J. Chem. Phys. 105, 2992 (1996).

[7] S. Watanabe, K. Kondo, Y. Nabekawa, A. Sagisaka, and Y. Kobayashi, Phys. Rev. Lett. 73, 2692 (1994).

[8] Y.-Y. Yin, C. Chen, and D.S. Elliott, Phys. Rev. Lett. 69, 2353 (1992).

[9] E. Papastathopoulos, D. Xenakis, and D. Charalambidis, Phys. Rev. A 59, 4840 (1999).

[10] H.-L. Kim and R. Bersohn, J. Chem. Phys. 107, 4546 (1997).
[11] D.W. Schumacher, F. Weihe, H.G. Miller, and P.H. Bucksbaum, Phys. Rev. Lett. 73, 1344 (1994).

[12] T. Nakajima and P. Lambropoulos, Phys. Rev. Lett. 70, 1081 (1993).

[13] T. Nakajima and P. Lambropoulos, Phys. Rev. A 50, 595 (1994).

[14] T. Nakajima, J. Zhang, and P. Lambropoulos, J. Phys. B 30, 1077 (1997).

[15] P. Lambropoulos and T. Nakajima, Phys. Rev. Lett. 82, 2266 (1999).

[16] K.J. Schafer and K.C. Kulander, Phys. Rev. A 45, 8026 (1992).

[17] E. Charron, A. Giusti-Suzor, and F.H. Mies, Phys. Rev. Lett. 71, 692 (1993).

[18] Y.-Y. Yin and D.S. Elliott, Phys. Rev. A 47, 2881 (1993).

[19] C.S. Feigerle, R.N. Compton, L.E. Cuéllar, N.A. Cherepkov, and L.V. Chernysheva, Phys. Rev. A 53, 4183 (1996).

[20] W. Ong and S.T. Manson, Phys. Rev. A 20, 2364 (1979). 